[3] Wallace ZS, Mattoo H, Mahajan VS, et al. Predictors of disease relapse in IgG4-related disease following rituximab. Rheumatology (Oxford). 2016 Jun;55(6):1000-8.

Disclosure of Interest: None declared

DOI: 10.1136/annrheumdis-2018-eular.3895

\section{THU0039 INSULIN-LIKE GROWTH FACTOR 1 RECEPTOR REGULATES THE PHENOTYPE AND FUNCTION OF CD21+ B CELLS}

M. Erlandsson, C. Wasen, G. Gravina, M.I. Bokarewa. Rheumatology and Inflammation Research, GOTHENBURG UNIVERSITY, Gothenburg, Sweden

Background: Ligand to the inducible T cell costimulator (ICOSL) on B cells is essential for the ICOS-dependent follicular recruitment of activated T cells. In patients with rheumatoid arthritis (RA) the IGF1-IGF1R axis is altered. Inhibition of IGF1R alleviated arthritis by reducing IL-6-dependent formation of Th17 cells. Here we study the role of IGF1R on CD21 +cells in experimental arthritis.

Methods: Female Balb/c mice were immunised with methylated BSA or with CII. Consequences of the IGF-1R inhibition for arthritis were studied in mBSA and CIIimmunised mice treated with NT157 compound promoting degradation of insulin receptor substrates or using shRNA producing construct (shIGF1R). At termination three sub-populations of CD21 +cells were analysed: follicular dendritic cells (FDc, CD21 +CD19-CXCR5-); marginal zone B cells (MZBc, CD21 +CD19 +CXCR5-); follicular B cells (FBc, CD21 +CD19+CXCR5+). Supernatants of LPSstimulated splenocytes were analysed for production of cytokines, chemokines using Cytokine Array. Serum levels of antigen specific and autoantibodies were measured in an ELISA.

Results: In spleen of mBSA-immunised mice, ICOSL expression on CD21 +cells correlated to IGF1R ( $r=0.70, p=0.007)$. Inhibition of IGF1R induced a $20 \%$ reduction in ICOSL expression in all CD21 +subsets $(p=0.007)$ followed by an increase in the number of MZBc ( $p=0.003)$, while FDc and FBc were unchanged. Inhibition of IGF1R had no effect on the expression of ICOS +on CD4 T cells or the subset of CXCR5 +follicular T cells. Reduction of the ICOSL +CD21+B cells was associated with lower production of IL-13. Inhibition of IGF1R signalling by NT157 and by shRNA, reduced production of CXCL13 and CXCL12, the chemokines essential for B cell migration towards follicules. In contrast, the production of chemokines CCL5 and CXCL12 preventing intra-follicular migration was increased, which explains the increase of MZBc. Additionally, the insufficient ICOSL signalling significantly reduced the production of IL-7 and IL-4, regulating class switching of B cells in germinal centres and differentiation of B cells into plasma cells. The described disbalance in the cytokines aiding $B$ cell development led to the reduced production anti-inflammatory $\mathrm{IL}-10$ and of mBSA-specific $\lg M(p=0.005)$ and increased production of autoreactive RF-IgM levels $(p=0.001)$

Conclusions: The study shows that IGF1R controls B cell development through the expression of ICOSL on CD21 +cells. Insufficient ICOSL signalling disturbs a balance between antigen-specific response and autoantibody production in experimental arthritis.

\section{REFERENCE:}

[1] Erlandsson MC, Toyra Silfversward S, Nadali M, Turkkila M, Svensson MND, Jonsson IM, et al. IGF-1R signalling contributes to IL-6 production and $T$ cell dependent inflammation in rheumatoid arthritis. Biochim Biophys Acta. 2017;1863:2158-70.

Disclosure of Interest: None declared

DOI: 10.1136/annrheumdis-2018-eular.5300

\section{THU0040 ENHANCEMENT OF EARLY HUMAN B CELL DEVELOPMENT BY JAK INHIBITION}

J. Thiel ${ }^{1}$, N. Venhoff ${ }^{1}$, I. Janowska ${ }^{1}$, A. Troilo ${ }^{1}$, N. Frede ${ }^{1}$, M. Erlacher ${ }^{2}$, M. Kunze ${ }^{3}$, R. Lorenzetti ${ }^{1}$, J. Staniek ${ }^{1}$, B. Bannert ${ }^{4}$, D. Kyburz ${ }^{4}$, C. Glaser ${ }^{1}$, M.-T. Schleyer ${ }^{1}$, R E. Voll ${ }^{1}$, M. Rizzi ${ }^{1} .{ }^{1}$ Rheumatology and Clinical Immunology, ${ }^{2}$ Clinic for Pediatrics and Juvenile Medicine; ${ }^{3}$ Gynecology Department, University Medical Center Freiburg, Freiburg, Germany; ${ }^{4}$ Department of Rheumatology, University of Basel, Basel, Switzerland

Background: Rheumatoid arthritis is an immune-mediated disease, in which immune cell activation leads to destructive inflammation of the joints. In this context the treatment with JAK inhibitor tofacitinib has been proven to be effective. In mice treatment with tofacitinib resulted in reduced specific antibody responses, failure to generate germinal centres, and partial block of B cell development in the bone marrow. Conversely in vivo treatment of psoriasis arthritis patients as well as of rheumatioid arthritis patients with tofacitinib results in an increase of relative and absolute numbers of B cells in the first 4-8 weeks from beginning of treatment. It is known that mouse early $B$ cell development is strongly dependent on IL-7 signalling, while this is not essential in humans. Nevertheless other cytokines are important in determining the fate and development of B lymphocytes. Hence, the outcome of JAK inhibition in early B cell development remains to be studied Objectives: To assess the impact of JAK inhibition on early B cell development in vitro

Methods: We used a in vitro model in which CD $34^{+}$cells isolated from cord blood are cultivated subsequently in SCF, Flt3-L and IL-6, then SCF, Flt3-L and IL-7 and finally in cytokine-free medium $\left(.^{1}\right.$ The culture reproduces all stages of develop ment from common lymphocytes progenitors to immature B cells.

Results: With the addition of tofacitinib to the in vitro culture we observed an increase in the absolute numbers of lymphoid precursors developing in vitro especially at week 5 and 6 of culture. Specifically, JAK inhibition led to an increase of pre-B and immature B cells by week 5 and 6 . These data are in contrast with the early $B$ cell development block observed in the tofacitinib treated mouse, but are in line with the rapid increase of B cells in peripheral blood after 4-8 weeks of tofacitinib treatment in patients. Analysis of induction of fate determining genes (EBF E2A, PAX-5) showed an earlier and stronger induction of fate determining genes. Conclusions: Our data indicate that JAK inhibition may promote early B cell development by enhancing the commitment of lymphoid precursors to the B cell compartment, contributing to a temporary increase in relative and absolute numbers of $B$ cell in peripheral blood of treated patients. These data contribute to our understanding of human B cell development, prompt us to further analyse the quality of $B$ cell output from the bone marrow in JAK inhibited patients, and may provide cues to understand the outcome of JAK inhibition treatment in rheumatic diseases.

\section{REFERENCE:}

[1] Kraus $\mathrm{H}$, et al. A feeder-free differentiation system identifies autonomously proliferating B cell precursors in human bone marrow. J Immunol. 2014 Feb 1;192(3):1044-54.

Disclosure of Interest: None declared

DOI: 10.1136/annrheumdis-2018-eular.5190

\section{THU0041 THE EFFECTS OF ABATACEPT ON HUMAN B CELL ACTIVITIES}

M.-H. Chen ${ }^{1}$, C.-T. Ssu ${ }^{2}$, C.-Y. Tsai ${ }^{1}$, C.-M. Leu ${ }^{3} .{ }^{1}$ Division of Allergy, Immunology and Rheumatology, Department of Medicine, Taipei Veterans General Hospital; ${ }^{2}$ Institute of Microbiology and Immunology, ${ }^{3}$ Institute of Microbiology and Immunology, and Infection and Immunity Center, National Yang-Ming University, Taipei City, Taiwan, Province of China

Background: Abatacept is a cytotoxic T lymphocyte antigen-4 (CTLA-4) fusion protein approved for rheumatoid arthritis (RA) treatment worldwide. Abatacept mimics the natural CTLA-4 and competes with CD28 for binding the CD80/CD86 on antigen presenting cells to prevent $T$ cell activation. However, the impacts of CTLA-4-lg through its interaction with CD80/CD86 on B cells are not fully understood.

Objectives: The aim of this study was to test whether CTLA-4 regulates human B cell functions

Methods: We assayed the effect of abatacept on human B cells in both in vitro and in vivo conditions. Blood was taken from 20 patients with RA before and after abatacept treatment and the expression of surface proteins on B cells was detected using immunofluorescent staining. Serum level of rheumatoid facto (RF) and anti-cyclic citrullinated peptide antibody (ACPA) was measured by ELISA. Purified human B cells from healthy donors were stimulated in the pres ence of abatacept and cell proliferation, cytokine production, plasma cell differentiation, and antibody production were measured.

Results: In RA patients, abatacept treatment transiently reduced the level of CD80/CD86 on peripheral blood memory B cells and increased the naïve to memory $\mathrm{B}$ cell ratio. Also, abatacept reduced lgM-RF level in 10 out of 12 patients, which correlates with patients' disease activity, but it had no significant effects on serum levels of anti-citrullinated protein antibody or anti-tetanus toxoid antibodies during the 6 months of abatacept treatment. In the in vitro assays, we observed that the CD80 and CD86 induced by T-independent (TI) but not T-dependent (TD) stimulation was significantly downregulated by abatacept at both the mRNA leve and protein level. Some Tl-induced cytokine production by B cells from healthy donors was also reduced by abatacept. Neither TI nor TD- stimulated B cell proliferation was reduced by abatacept in ${ }^{3} \mathrm{H}$-thymidine incorporation assay. Abatacept had no significant effect on CD38 ${ }^{\text {high }} \mathrm{CD} 27^{\text {high }}$ plasma cell differentiation. Finally abatacept inhibited Daudi-B cell induced allogeneic T cell proliferation, indicating a significant blockade of T-B interaction by abatacept.

Conclusions: In RA patients, abatacept may decrease RF level by interfering the interaction of CD28 with CD80/CD86, therefore preventing B cells from T cells' 